\title{
Sound Wave Sterilization
}

National Cancer Institute

\section{Source}

National Cancer Institute. Sound Wave Sterilization. NCI Thesaurus. Code C101695.

A sterilization process that uses high-frequency sound waves to kill microorganisms. 